\title{
Micromechanical Origin of Particle Size Segregation
}

\author{
L. Jing and C. Y. Kwok \\ Department of Civil Engineering, The University of Hong Kong, Pokfulam Road, Hong Kong, China \\ Y. F. Leung \\ Department of Civil and Environmental Engineering, The Hong Kong Polytechnic University, Hong Kong, China
} (Received 25 May 2016; revised manuscript received 13 December 2016; published 15 March 2017)

\begin{abstract}
We computationally study the micromechanics of shear-induced size segregation and propose distinct migration mechanisms for individual large and small particles. While small particles percolate through voids without enduring contacts, large particles climb under shear through their crowded neighborhoods with anisotropic contact network. Particle rotation associated with shear is necessary for the upward migration of large particles. Segregation of large particles can be suppressed with inadequate friction, or with no rotation; increasing interparticle friction promotes the migration of large particles, but has little effect on the percolation of small particles.
\end{abstract}

DOI: 10.1103/PhysRevLett.118.118001

Flows of granular materials under shear or vibration, whose constituents may differ in size [1-7], density [8-10], surface roughness [11], and inelasticity [12], tend to segregate into each species. The understanding of such segregation is of great importance to industrial processes, e.g., for the quality control of granular products [13-15]. In geophyiscal granular flows, segregation often leads to selfchannelized morphology and enhanced run-out distance [16-20]. Over decades, continuum theoretical models of segregation [21-25] have evolved with significant insights added from grain-scale measurements in laboratory experiments [7] and computer simulations [26]. For shear-induced size segregation, small particles fall through the local voids opened by shear (i.e., kinetic sieving), and large particles drift toward the surface due to imbalanced contact force (i.e., squeeze expulsion) [21]. While kinetic sieving is well recognized in many contexts, the mechanics related to squeeze expulsion remain ambiguous. Little evidence has been provided from physical experiments due to the difficulty in accessing grain-scale information, especially the contact force inside flowing materials [7,27-30]. On the other hand, numerical experiments, such as those conducted using the discrete element method, can produce detailed information of velocity, volume fraction, and contact force [31-37]. Statistical analysis of such information can enhance the understanding of the micromechanical origin of squeeze expulsion, which is the major goal of this work.

In our simulation setup, periodic boundaries are imposed to the streamwise $(x)$ and spanwise $(y)$ directions, representing an infinitely wide and long chute [38]. The base of the chute is roughened by fixing a random packing of small particles [39]; the top surface is free. At the initial state [Fig. 1(a)], a layer of small particles (diameter $d_{s}$ ) is placed on top of a bed of large particles (diameter $d_{l}$ ), which we refer to as normal grading (NG). The initial flow thickness is $H_{0}$, and the volume fraction of large particles is defined as $\Phi_{l}=V_{l} /\left(V_{l}+V_{s}\right)$, where $V_{l}$ and $V_{s}$ are total volumes of large and small particles, respectively. To initiate the flow, gravity is tilted to a target inclination $\theta$ in the $x z$ plane. Unless otherwise specified, the following configuration [40] is adopted: $d_{s}=0.005 \mathrm{~m}, d_{l}=0.01 \mathrm{~m}, H_{0}=40 d_{s}$, $\Phi_{l}=0.5$, and $\theta=25^{\circ}$. Particle properties include density $\rho=2650 \mathrm{~kg} / \mathrm{m}^{3}$, Young's modulus $E=10^{9} \mathrm{~Pa}$, and Poisson's ratio $\nu=0.35$. The contact force is calculated using the Hertz model [38], where normal damping is given by $\gamma_{n}=\ln (e) / \Delta t ; e=0.8$ is the coefficient of restitution and $\Delta t=10^{-5} \mathrm{~s}$ is collision time. No tangential damping is considered. The Coulomb friction criterion is applied, i.e., $\left|f_{t}\right| \leq\left|\mu f_{n}\right|$, where $\mu=0.5$ is the coefficient of friction; $f_{n}$ and $f_{t}$ are normal and tangential contact forces, respectively.

After the flow is initiated under gravity, mixing and segregation emerge subsequently. In Fig. 1(b), segregation reaches a final equilibrium and a layer of large particles reaches the surface. The interface of the two species is blurred (due to diffusive remixing [23]) and a number of large particles remain immersed in the "sea" of small
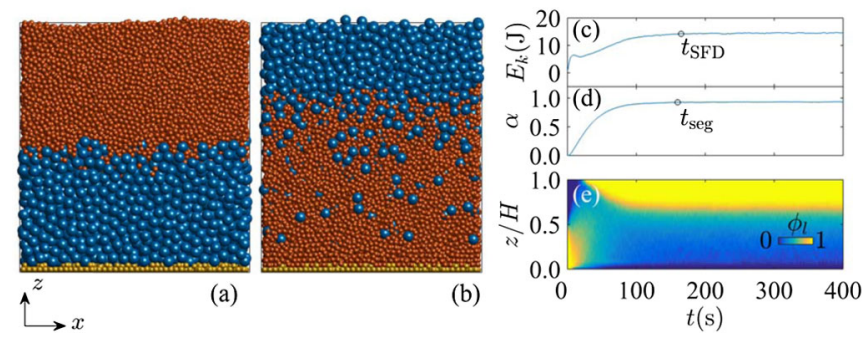

FIG. 1. Basic information. A bidisperse flow $\left(\Phi_{l}=0.5\right)$ at the (a) initial and (b) final state. The temporal evolution of (c) kinetic energy $E_{k}$, (d) the degree of segregation $\alpha$, and (e) the distribution of local concentration of large particles $\left(\phi_{l}\right)$. 
particles (to be discussed later). To describe the degree of segregation, we define $\alpha=\left(1-\Delta c / \Delta c_{0}\right) / 2$, where $\Delta c=c_{s}-c_{l}$ is the distance between the centers of mass of small $\left(c_{s}\right)$ and large $\left(c_{l}\right)$ particles, and $\Delta c_{0}$ is the initial distance. With this definition, $\alpha=0$ is the initial state and $\alpha=1$ represents perfect segregation. In Figs. 1(c) and 1(d), we compare the temporal developments of kinetic energy $\left(E_{k}\right)$ and the degree of segregation $(\alpha)$. The process of segregation generally corresponds to the flow dynamics. The flow becomes steady, fully developed (SFD) [31] after $t_{\mathrm{SFD}}=165 \mathrm{~s}$, while segregation completes after $t_{\mathrm{seg}}=160 \mathrm{~s}$, with a final degree of segregation $\alpha_{f} \approx 0.92$. Figure 1(e) presents a detailed evolution of the specific distribution of large particles and confirms that the state of imperfect segregation sustains in the SFD state.

Our results [40] show that $\alpha_{f}$ is relatively insensitive to $\theta \in[22,30]^{\circ}$ (where a SFD state is reached) and $\Phi_{l} \in[0.4,0.8]$. Beyond the moderate range of $\Phi_{l}, \alpha_{f}$ gets closer to 1 [see Fig. 2(c) inset]. A similar conclusion is drawn in [36] that $\theta$ mainly changes the segregation rate but not the maximum extent of segregation. Here we statistically examine the segregation displacement $\Delta z / H$ for large and small particles, where $H$ is the evolving flow thickness. We propose that upon perfect segregation (i.e., $\alpha_{f}=1$ ), the theoretical probability distribution function (PDF) of $\Delta z / H$ for the constituent $I(I=l, s$ represents large or small particles) obeys a normal distribution-the magnitude of its mean equals the distance traveled by the center of mass, i.e., $|\bar{\Delta} z / H|=1-\Phi_{I}(\overline{\Delta z}$ is positive when $I=l$ and negative otherwise); the PDF is confined by $1-2 \Phi_{I} \leq|\Delta z / H| \leq 1$. The first inequality means that a particle travels the shortest distance if it infiltrates back through its own species (whose thickness is approximately $\left.\Phi_{I} H\right)$, and the second inequality indicates that the maximum travel distance is the flow thickness $H$. For imperfect segregation $\left(\alpha_{f}<1\right)$, the actual PDF is expected to deviate from this theoretical shape.

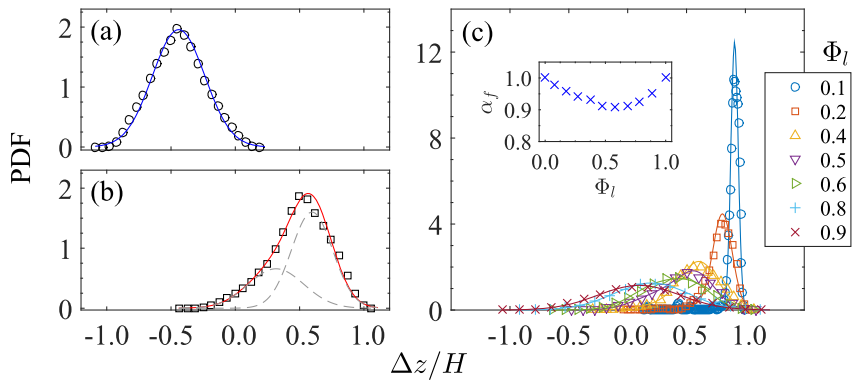

FIG. 2. Displacement statistics. (a) PDF for small particles in $\Phi_{l}=0.5$. The solid line is fitted by a normal distribution. (b) PDF for large particles in $\Phi_{l}=0.5$. The solid line is fitted by a Gaussian mixture distribution, with dashed lines showing the two components. (c) PDFs for large particles with $\Phi_{l}$ varying in [0.1, $0.9]$. Lines are fitted by Gaussian mixture distributions. Inset: $\alpha_{f}$ as a function of $\Phi_{l}$.
Figures 2(a) and 2(b) present the PDFs for large and small particles, respectively, in the case $\Phi_{l}=0.5$. A normal distribution is found for small particles (with skewness $s=0.088)$, which is centered approximately at $-\left(1-\Phi_{s}\right)=$ -0.5 and bounded by -1 and $2 \Phi_{s}-1=0$. The good normality confirms the random movements of small particles relative to their center of mass. The small skewness to the right is a result of diffusive remixing. For large particles, the PDF is significantly skewed $(s=-0.49)$ with a long tail to the left, which can be fitted by a Gaussian mixture distribution [Fig. 2(b)]. We attribute these features to the mixing at the interface and the large particles stuck in the sea of small particles [Fig. 1(b)]. Despite the high skewness, the peak of the PDF locates approximately at $1-\Phi_{l}=0.5$ with the upper bound at around 1 .

We then explore the PDFs of large particles as $\Phi_{l}$ varies from 0.1 to 0.9. As seen in Fig. 2(c), all PDFs are centered approximately at $1-\Phi_{l}$, and bounded by $1-2 \Phi_{l}$ and 1 . In the moderate range of $\Phi_{l}$, the similar skewness reflects the almost unaltered $\alpha_{f}$ in the inset of Fig. 2(c). When the population of large particles is small (e.g., $\Phi_{l}=0.1$ ), only a few of them remain in mixing. If large particles are the majority (e.g., $\Phi_{l}=0.9$ ), they can easily climb up the small ones. Furthermore, we investigate the extreme cases of only one single large $\left(\Phi_{l} \rightarrow 0\right)$ or small particle $\left(\Phi_{l} \rightarrow 1\right)$ as an intruder. In both cases, the single particle travels all the way through its opponents, i.e., $\alpha_{f}=1$, which leads to the $U$-shape trend in the inset of Fig. 2(c).

The "entrapment" of large particles is a major source of imperfect segregation, as discussed above. Although commonly reported in bidisperse chute flows [35-37,40], it may seem counterintuitive because even a single large particle can eventually migrate to the top. Here we track the trajectories of these unsegregated particles and find out that they are in fact not stagnant: the lower layers exchange large particles with the top layers, with a dynamic equilibrium established. The equilibrium should be more stable than the state of perfect segregation. To confirm this, we change the initial setup to reverse grading $(\mathrm{RG})$ where large particles stay initially atop the small ones, and mixture (MX) where the two species are thoroughly mixed before flowing. All three setups lead to the same state of segregation [Fig. 3(a)] with an identical final distribution of large particles [Fig. 3(b)]. Unlike the symmetric interfacial mixing of large and small particles predicted by the theory of diffusive remixing [23], large particles propagate further toward the bottom than small particles toward the surface [Fig. 1(b)]. Such an asymmetric distribution of large and small particles may result from either skewed segregation flux [7,24] or unequal coefficients of diffusivity. Further investigation is required to take this into theoretical consideration.

The highly skewed PDF of displacement in large particles (Fig. 2) also implies a fundamental difference in the movement mechanism of large and small particles, which should have its origin at the particle scale. Here we 

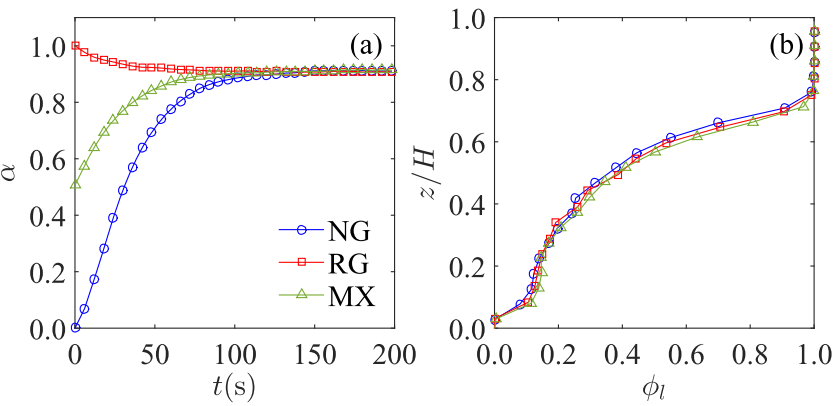

FIG. 3. Saturation of segregation. (a) Evolution of $\alpha$ for NG, $\mathrm{RG}$, and MX. (b) Local concentration of large particles $\phi_{l}$ at the final steady state.

explore the micromechanics of the flow, including its coordination number $(Z)$, particle-based connectivity $\left(Z_{i}\right)$, and contact network. As shown in Fig. 4(a), $Z$ bifurcates as the two species are mixed. Compared to a control setup of monodisperse flow (particle diameter $d_{s}$ ), small particles have much lower $Z$ as they are accommodated in the voids through large particles, while large
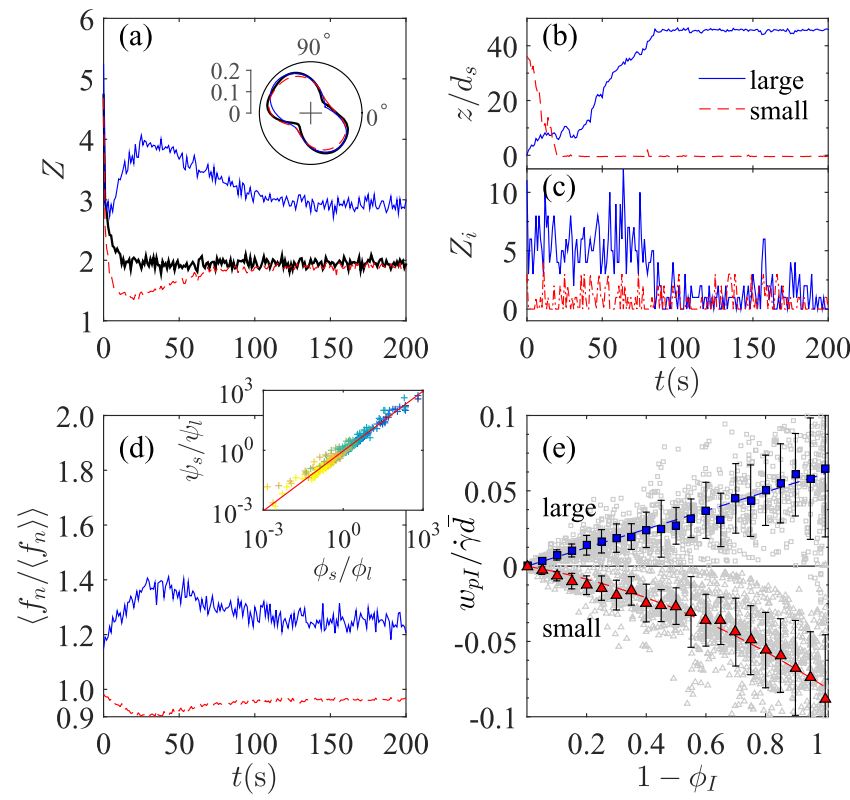

FIG. 4. Microstructures. (a) Evolution of coordination number $Z$ for large (solid, blue) and small (dashed, red) particles and a control setup of monodisperse flow (thick black). Inset: contact orientation. (b) Trajectory and (c) connectivity $Z_{i}$ of a single large $\left(\Phi_{l} \rightarrow 0\right.$, blue) and a single small $\left(\Phi_{l} \rightarrow 1\right.$, red) particle. (d) Evolution of the average normal contact force, $\left\langle f_{n} /\left\langle f_{n}\right\rangle\right\rangle$, for large (solid, blue) and single small (dashed, red) particles. Inset: stress partitioning for $\Phi_{l}$ varying from 0.1 (dark markers) to 0.9 (bright markers). The red solid line is a linear fit in log scale with slope 0.9260 . (e) Percolation velocity $w_{p I} / \dot{\gamma} \bar{d}$ as a function of local concentration $1-\phi_{I}(I=l, s)$. Blue squares and red triangles (with error bars) are the mean values of raw data (gray points) for large and small particles, respectively. Dashed lines are fitted with the means. particles experience a dramatic increase in $Z$ as small particles occupy the voids surrounding them. After segregation, $Z$ gets steady [37,41] but remains high in large particles due to the interfacial mixing. In Figs. 4(b) and 4(c), the trajectories and connectivities of a large particle and a small particle are presented simultaneously. The dropping of the small particle is much faster than the climbing of the large particle, which is consistent with the asymmetric segregation flux proposed in [7].

With the number of contacts distinguished between large and small particles during segregation, we are interested in the distribution of these contacts and the magnitude of contact forces. The orientation of the contact network in the $x z$ plane is delineated as rose diagrams [Fig. 4(a) inset], which exhibit an inherent shear-induced anisotropy $[41,42]$. We observe no difference between large and small particles, meaning that the contact orientation is statistically insensitive to the constituent grain size. Figure 4(d) presents the evolution of normal contact force, $f_{n}$, which is normalized by the average force at a specific elevation, $\left\langle f_{n}\right\rangle$, to eliminate the differences caused by gravity $[37,43,44]$. Besides the high connectivity experienced by large particles, they carry much higher contact forces than small particles. From the perspective of continuum mixture theory for bidisperse flows [22,23], large particles rise if the proportion of pressure they carry is higher than the proportion of volume they occupy. Let $\psi_{I}$ be the proportion of pressure on constituent $I(I=l, s)$; a simple form proposed in [22] is $\psi_{I}=\phi_{I}\left[1 \pm B\left(1-\phi_{I}\right)\right]$, where the \pm sign is taken as positive when $I=l$ and negative otherwise; $B$ is a nondimensional perturbation away from volume concentration $\phi_{I}$. In [22], the percolation velocity of species $I, w_{p I}$, is given by

$$
w_{p I}= \pm(B / C) g \cos \theta\left(1-\phi_{I}\right)
$$

where $C>0$ is the drag coefficient, and $g$ is the gravitational acceleration. Equation (1) predicts segregation (i.e., large particles rise and small particles drop) only if $B>0$. We verify the value of $B$ during segregation (at a $10 \mathrm{~s}$ period with fastest segregation) for cases $\Phi_{l} \in[0.1,0.9]$, following the stress partitioning suggested in [37]. For each case, all local variables are calculated in layers with $2 d_{s}$ in thickness. In the inset of Fig. 4(d), we plot $\psi_{s} / \psi_{l}$ against $\phi_{s} / \phi_{l}$ and the linear fitting yields $B=0.08$. The positive value of $B$ supports that the excess pressure taken by large particles drives them to rise.

Equation (1) shows a clear correlation between $w_{p I}$ and $1-\phi_{I}$. Note that the theory in [23] adds $-D \partial\left(\ln \phi_{I}\right) / \partial z$ to the right-hand side of Eq. (1) to consider diffusion, where $D>0$ is diffusivity. The additional term has a negative sign because diffusion tends to remix particles along the concentration gradient. Since the determination of parameters $C$ and $D$ is nontrivial in the current transient system, we only correlate $w_{p I}$ with $1-\phi_{I}$ as implied by Eq. (1). 
Figure 4(e) shows the correlation for all cases with the overall concentration $\Phi_{l} \in[0.1,0.9]$, in which $w_{p I}$ is normalized by a local velocity scale $\dot{\gamma} \bar{d}$ [45]. A tendency exists that when one constituent is more immersed in its opponent, it can gain a higher magnitude of percolation velocity. Similar correlations have been found in the segregation of steady flows $[21,23,45]$. Notice that the correlation in Fig. 4(e) is smeared, which is attributed to two reasons: First, the process of segregation is transient in the current system [Fig. 1(d)] and the fluctuation of velocity is intrinsic in shear flows $[38,41,46]$. Secondly, diffusion might hinder the local rate of segregation [23].

Our next focus is the migration mechanism of individual large and small particles during segregation. Snapshots of the flow with $\Phi_{l}=0.5$ are taken at a high frequency of $1000 \mathrm{~Hz}$. We trace one focused large (or small) particle while it is continuously rising (or dropping) in a time window of $5 \mathrm{~ms}$ at $t=10 \mathrm{~s}$; its close neighborhood is analyzed (see [47] for two videos showing these processes). In Fig. 5(a), the small particle resides in the void of large particles and penetrates easily under gravity. More instantaneous contacts are observed than durable ones. This explains the fast dropping of a single particle in Fig. 4(b). Around the large particle [Fig. 5(b)], a crowded neighborhood is observed as small particles fill the voids. The contact network is anisotropic and the orientation coincides with the rose diagram in the inset of Fig. 4(a). The geometric anisotropy implies a strong barrier against the movement along contact orientation [28]. Furthermore, a tendency of shear is observed as the upper neighbors flow faster than the lower ones. This velocity gradient is inherent in surface-driven shear flows (such as [38]). As a result, the focused particle is under enduring shear while struggling out of the current position. The anisotropic contact network and the shear due to velocity gradient constitute the mechanism of squeeze expulsion [21]. The migration of a large particle is more stumbling than its small counterpart [Fig. 4(b)], since the possibility of finding voids and being squeezed downward is inevitable. (a)

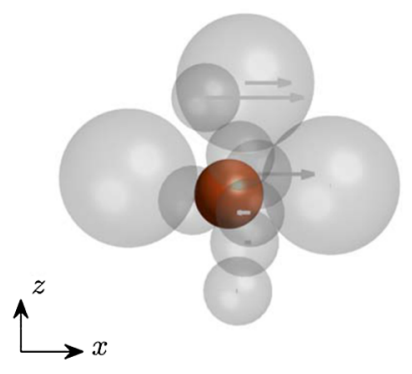

(b)

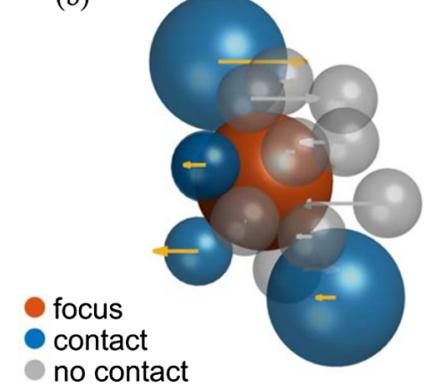

FIG. 5. Migration mechanism. The neighborhoods of (a) a dropping small particle and (b) a climbing large particle, in the flow of $\Phi_{l}=0.5$. Arrows represent velocities relative to the focused particle.
The linkage between shear movements and squeeze expulsion implies that the segregation of large particles should be more significantly affected by interparticle friction. To test the role of friction without disturbing the flow kinetics, we simulate single-intruder cases and only vary the coefficient of friction between large and small particles, i.e., $\mu_{l s} \in[0.1,1.0]$. Similar to the trajectories in Fig. 4(b), a large intruder climbs toward the free surface, while a small intruder drops toward the base. For each value of $\mu_{l s}$, we perform six independent runs and measure the mean time of segregation $t_{\text {seg }}$. As shown in Fig. 6(a), $t_{\text {seg }}$ for the small particle is insensitive to friction, which is expected as the small particle is free from shear. For the large particle, a significant impact of $\mu_{l s}$ is observed on the value of $t_{\text {seg }}$, which supports the proposition that large particles are subject to shear during squeeze expulsion. When $\mu_{l s}$ is low, it takes much longer for the intruder to complete segregation. In fact, segregation is substantially suppressed with inadequate frictional strength (segregation cannot complete within $2000 \mathrm{~s}$ for $\mu_{l s} \leq 0.1$ ). Since $\mu_{l s}$ limits the maximum shear force allowed at a contact before sliding occurs, lower $\mu_{l s}$ indicates less chance of rotation. As such, we propose that rotation is necessary for the migration of large particles. Indeed, as we impose an arbitrarily high rolling resistance [48] to the large intruder, no segregation can occur. Furthermore, in the bidisperse mixture with $\Phi_{l}=0.5$, the change of bulk friction $\mu$ has a similar effect on the final degree of segregation $\alpha_{f}$ [Fig. 6(b)]. Reduction of $\mu$ leads to a significant drop of $\alpha_{f}$, and higher $\mu$ promotes more large particles to reach the top. Further investigation is needed to take into account the role of friction from the theoretical perspective, such as to consider whether large particles undergo interphase drag [23] in a different way than small particles.

To conclude, we explain the origin of preferential upward movements of large particles. We link the high connectivity in segregating large particles to the higher contact force they carry; it is equivalent to the excess pressure on large particles in the continuum theory, which acts as the driving mechanism of upward percolation velocity. In addition, we propose a mechanism for the upward migration of large particles, which is fundamentally different from the percolation of
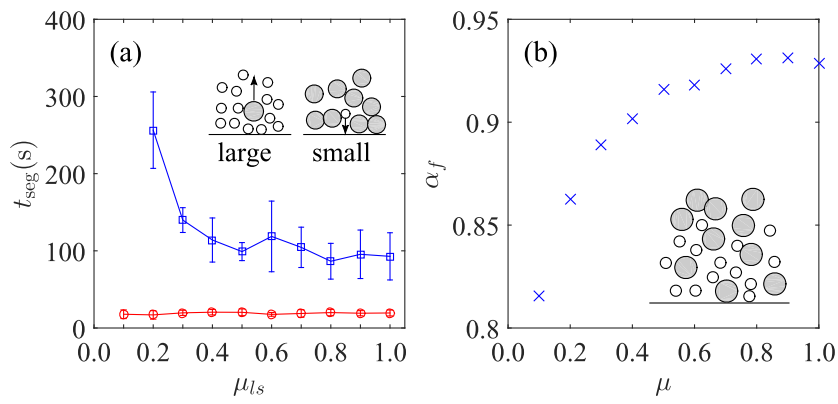

FIG. 6. Role of friction. (a) Single intruder cases. Square markers: large intruder. Circular markers: small intruder. (b) $\Phi_{l}=0.5$ cases. 
small particles: they are subject to enduring shear while struggling out of their crowded, anisotropic neighborhood. This work is in line with attempts toward a theoretical framework of granular segregation, where the underlying asymmetric behavior of large and small particles has been recently incorporated $[22-25,35,45]$. Our findings regarding the saturation of segregation, the distinct migration mechanisms in large and small particles, and the role of friction in segregation are expected to contribute to the framework.

The work was supported by Research Grants Council of Hong Kong (Grant No. 17203614) and the Research Institute for Sustainable Urban Development at the Hong Kong Polytechnic University. This research is conducted in part using the research computing facilities and advisory services offered by Information Technology Services, the University of Hong Kong.

*fiona.kwok@hku.hk

[1] J. Duran, J. Rajchenbach, and E. Clement, Phys. Rev. Lett. 70, 2431 (1993).

[2] J. B. Knight, H. M. Jaeger, and S. R. Nagel, Phys. Rev. Lett. 70, 3728 (1993).

[3] O. Zik, D. Levine, S. G. Lipson, S. Shtrikman, and J. Stavans, Phys. Rev. Lett. 73, 644 (1994).

[4] O. Pouliquen, J. Delour, and S. B. Savage, Nature (London) 386, 816 (1997).

[5] K. M. Hill and Y. Fan, Phys. Rev. Lett. 101, 088001 (2008).

[6] M. Harrington, J. H. Weijs, and W. Losert, Phys. Rev. Lett. 111, 078001 (2013).

[7] K. van der Vaart, P. Gajjar, G. Epely-Chauvin, N. Andreini, J. M. N. T. Gray, and C. Ancey, Phys. Rev. Lett. 114, 238001 (2015).

[8] D. C. Hong, P. V. Quinn, and S. Luding, Phys. Rev. Lett. 86, 3423 (2001).

[9] A. P. J. Breu, H.-M. Ensner, C. A. Kruelle, and I. Rehberg, Phys. Rev. Lett. 90, 014302 (2003).

[10] D. A. Huerta and J. C. Ruiz-Suarez, Phys. Rev. Lett. 92, 114301 (2004).

[11] K. A. Gillemot, E. Somfai, and T. Borzsonyi, Soft Matter 13 (2017), 415.

[12] C. R. K. Windows-Yule, T. Weinhart, D. J. Parker, and A. R. Thornton, Phys. Rev. Lett. 112, 098001 (2014).

[13] H. A. Makse, S. Havlin, P. R. King, and H. E. Stanley, Nature (London) 386, 379 (1997).

[14] J. M. Ottino and D. V. Khakhar, Annu. Rev. Fluid Mech. 32, 55 (2000).

[15] T. Shinbrot and F. J. Muzzio, Phys. Today 53, 25 (2000).

[16] G. Felix and N. Thomas, Earth Planet. Sci. Lett. 221, 197 (2004).

[17] M. Kleinhans, Earth-Sci. Rev. 65, 75 (2004).

[18] C. G. Johnson, B. P. Kokelaar, R. M. Iverson, M. Logan, R. G. LaHusen, and J. M. N. T. Gray, J. Geophys. Res. 117 (2012).

[19] B. Kokelaar, R. Graham, J. Gray, and J. Vallance, Earth Planet. Sci. Lett. 385, 172 (2014).
[20] T. de Haas, L. Braat, J. R. F. W. Leuven, I. R. Lokhorst, and M. G. Kleinhans, J. Geophys. Res. 120, 1949 (2015).

[21] S. B. Savage and C. K. K. Lun, J. Fluid Mech. 189, 311 (1988).

[22] J. M. N. T. Gray and A. R. Thornton, Proc. R. Soc. A 461, 1447 (2005).

[23] J. M. N. T. Gray and V. A. Chugunov, J. Fluid Mech. 569, 365 (2006).

[24] J. M. N. T. Gray and C. Ancey, J. Fluid Mech. 779, 622 (2015).

[25] P. Gajjar, K. van der Vaart, A. R. Thornton, C. G. Johnson, C. Ancey, and J. M. N. T. Gray, J. Fluid Mech. 794, 460 (2016).

[26] Y. Fan and K. M. Hill, Phys. Rev. Lett. 106, 218301 (2011).

[27] Y. Fan, Y. Boukerkour, T. Blanc, P. B. Umbanhowar, J. M. Ottino, and R. M. Lueptow, Phys. Rev. E 86, 051305 (2012).

[28] T. S. Majmudar and R. P. Behringer, Nature (London) 435, 1079 (2005).

[29] E. I. Corwin, H. M. Jaeger, and S. R. Nagel, Nature (London) 435, 1075 (2005).

[30] M. van Hecke, Nature (London) 435, 1041 (2005).

[31] R. Delannay, M. Louge, P. Richard, N. Taberlet, and A. Valance, Nat. Mater. 6, 99 (2007).

[32] A. Tripathi and D. V. Khakhar, Phys. Fluids 23, 113302 (2011).

[33] A. Thornton, T. Weinhart, S. Luding, and O. Bokhove, Int. J. Mod. Phys. C 23, 1240014 (2012).

[34] Y. Fan, C. P. Schlick, P. B. Umbanhowar, J. M. Ottino, and R. M. Lueptow, J. Fluid Mech. 741, 252 (2014).

[35] D. R. Tunuguntla, O. Bokhove, and A. R. Thornton, J. Fluid Mech. 749, 99 (2014).

[36] L. Staron and J. C. Phillips, Phys. Fluids 26, 033302 (2014).

[37] L. Staron and J. C. Phillips, Phys. Rev. E 92, 022210 (2015).

[38] L. E. Silbert, D. Ertas, G. S. Grest, T. C. Halsey, D. Levine, and S. J. Plimpton, Phys. Rev. E 64, 051302 (2001).

[39] L. Jing, C. Y. Kwok, Y. F. Leung, and Y. D. Sobral, Phys. Rev. E 94, 052901 (2016).

[40] L. Jing, C. Y. Kwok, and Y. F. Leung, in International Conference on Particle-Based Methods, Fundamentals and Applications, PARTICLES 2015, Barcelona, Spain, 2015, edited by E. Onate, M. Bischoff, D. R. J. Owen, P. Wriggers, and T. Zohdi (International Center for Numerical Methods in Engineering (CIMNE), Barcelona, 2015).

[41] F. da Cruz, S. Emam, M. Prochnow, J.-N. Roux, and F. Chevoir, Phys. Rev. E 72, 021309 (2005).

[42] E. Azema and F. Radjai, Phys. Rev. Lett. 112, 078001 (2014).

[43] D. M. Mueth, H. M. Jaeger, and S. R. Nagel, Phys. Rev. E 57, 3164 (1998).

[44] D. M. Mueth, G. F. Debregeas, G. S. Karczmar, P. J. Eng, S. R. Nagel, and H. M. Jaeger, Nature (London) 406, 385 (2000).

[45] C. P. Schlick, Y. Fan, A. B. Isner, P. B. Umbanhowar, J. M. Ottino, and R. M. Lueptow, AIChE J. 61, 1524 (2015).

[46] O. Pouliquen, Phys. Rev. Lett. 93, 248001 (2004).

[47] See Supplemental Material at http://link.aps.org/ supplemental/10.1103/PhysRevLett.118.118001 for two videos showing a rising large particle and a sinking small particle, respectively.

[48] J. Ai, J.-F. Chen, J. M. Rotter, and J. Y. Ooi, Powder Technol. 206, 269 (2011). 